\title{
Polyimide 辐照效应的紫外及可见光谱研究
}

\author{
许 东 徐兴龙 林梓金 杜根娣 邹世昌 \\ (中国科学院上海治金研究所,上海 200050)
}

\section{关链词聚酰亚胺、离子注人、紫外及可见光橧}

聚酰证胺（polyimide）是一种电绝缘特性极好的热稳定芳香族聚合物，也被用作光致抗 蚀剂. 现已发现经过适当剂量的离子注人之后, 聚酰亚胺的电导率能提高约 20 个量级, 达到 半导体水平 ${ }^{[1]}$. 作为抗电磁辐射封装材料和温敏材料, 聚酰亚胺已受到广泛嚁目, 被公认是一 种值得重视的新型功能材料. 近年来的研究已经表明：聚酰亚胺改性层电导率的提高与其内 部类石墨相的形成存在密切的联系 ${ }^{[2-4]}$. 本文通过紫外及可见光谱研究了注入过程中聚酰亚 胺的结构变化, 以期对其中类石墨相的形成有一个较深人的了解.

\section{1 实验}

在经过清洗的双面抛光的石英片上用旋涂 (spinning coating)的方法涂一层聚酰亚胺酸 膜, 厚度控制在 $0.3 \mu \mathrm{m}$ 左右, 在烘箱内进行了亚胺化处理后就可得到聚酰亚胺薄膜. 这里所 制备的改性膜均采用 $120 \mathrm{keV}$ 的唧离子, 在 $1 \mu \mathrm{A} / \mathrm{cm}^{2}$ 的束流强度和室温( $\mathrm{RT}$ )下注人, 注入剂 量从 $1 \times 10^{14}$ 到 $1 \times 10^{16} \mathrm{~B}^{+} / \mathrm{cm}^{2}$. 紫外及可见光谱是在 Shimadzu UV-240 型紫外及可见分 光度计上得到的, 测试范围从 $200-800 \mathrm{~nm}$.

\section{2 结果与讨 论}

图 1 给出了一系列不同剂量注入样品的紫外及可见透射光谱. 可以看到透射率曲线随波 长增加而迅速上升, 呈现出类似于 $\mathrm{a}-\mathrm{C}: \mathrm{H}$ 的吸收边; 但是随着离子注人剂量的增加, 吸收边 逐渐移向红外波段一侧, 而且越来越不明显. 所有样品在低于 $300 \mathrm{~nm}$ 的波段内都完全被吸 收, 这里没有看到芳香环的吸收峰主要是因为薄膜过厚. 由于注入后的聚酰亚胺呈高度无序 状态, 因此可用无定形半导体中的 Tauc 公式以 以更加准确地定出吸收边 $E_{\mathrm{g}}$ :

$$
(\alpha E)^{1 / 2}-E-E_{g} \text {, }
$$

其中 $\alpha$ 是吸收系数, $E$ 是光子能量, $E g$ 即为对应光吸收边的能隙. 因此通过 Tauc 公式我 们可从紫外及可见光谱的线性拟合与外推中得到 $E_{z}$. 图 2 是各个注人剂量不同的样品的 $E_{8}$ 值, 可以看到随着剂量从 $1 \times 10^{14} \mathrm{~B}^{+} / \mathrm{cm}^{2}$ 增加到 $1 \times 10^{15} \mathrm{~B}^{+} / \mathrm{cm}^{2}, E_{z}$ 逐渐从 $1.4 \mathrm{eV}$ 减 至 $0.9 \mathrm{eV}$, 请注意在 $1 \times 10^{44} \mathrm{~B}^{+} / \mathrm{cm}^{2}$ 增至 $2 \times 10^{14} \mathrm{~B}^{+} / \mathrm{cm}^{2}$ 的过程中 $E_{\mathrm{g}}$ 反而略有上升, 这主 要是我们在实际应用 (1) 式确定 $E_{g}$ 时, 为方便起见假定了各个注人剂量下的薄膜厚度相同, 于是直接用吸光度代替了吸收系数, 但事实上在注人过程刚开始时, 薄膜厚度是有变化的的. 当剂量进一步增至 $1 \times 10^{15} \mathrm{~B}^{+} / \mathrm{cm}^{2}, E_{\text {z }}$ 则跌至不足 $0.1 \mathrm{eV}$, 能隙几乎消失. Robertson 等人

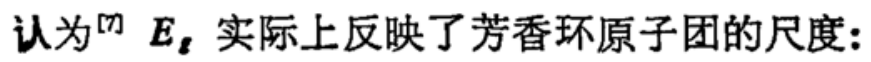

$$
E_{\varepsilon}=2 \beta / M \text {, }
$$

1992-04-20 收稿, 1992-10-28收修改稿。 


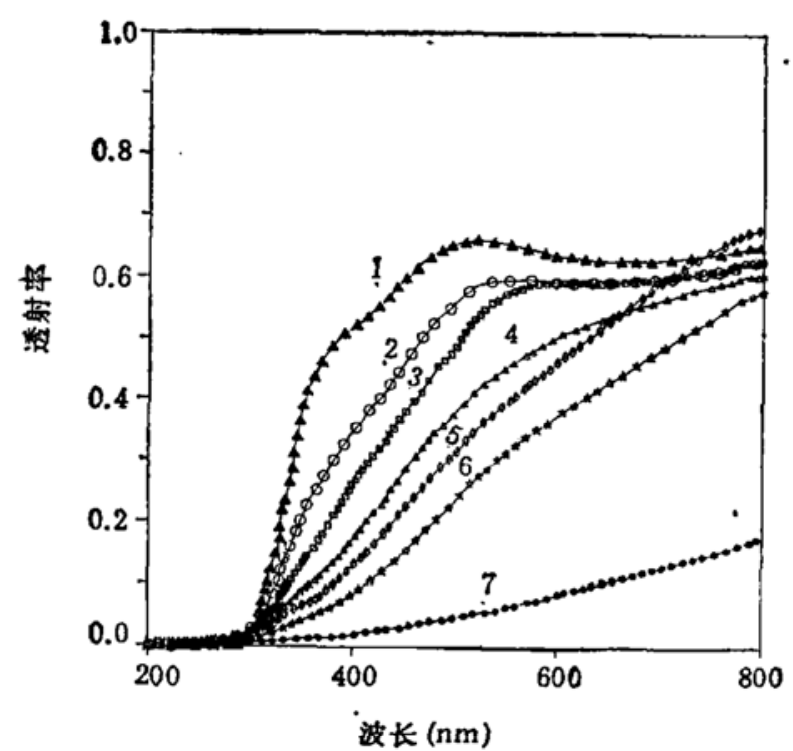

图 1 不同注入剂量聚酰亚胺薄膜的紫外及可见透射光谱可 以看到光吸收边随离子注入剂最的增加而逐渐移向红外波段 1 为末注人, 2 为 $1 \times 10^{14} \mathrm{~B}^{+} / \mathrm{cm}^{2}, 3$ 为 $2 \times 10^{14} \mathrm{~B}^{+} / \mathrm{cm}^{2}, 4$ 为 $4 \times 10^{14} \mathrm{~B}^{+} / \mathrm{cm}^{2}, 5$ 为 $8 \times 10^{14} \mathrm{~B}^{+} / \mathrm{cm}^{2}, 6$ 为 $1 \times 10^{15} \mathrm{~B}^{+} / \mathrm{cm}^{2}, 7$ 为 $1 \times 10^{16} \mathrm{~B}^{+} / \mathrm{cm}^{2}$

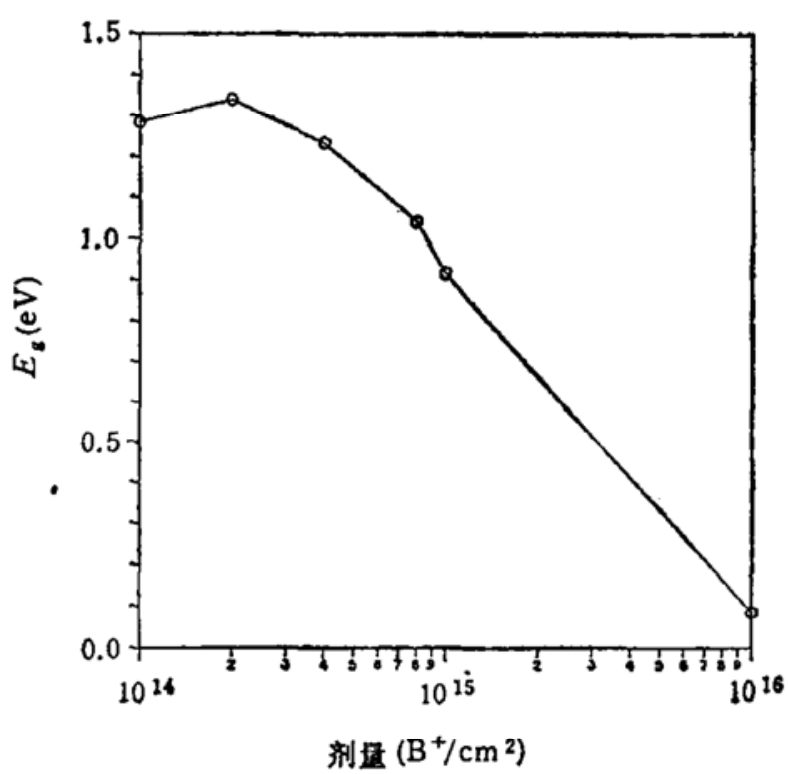

图 2 经离子轰击聚酰亚胺的光吸收边 $E_{g}$ (亦即能隙) 随注入剂量的变化 $E_{z}$ 是用 $\mathrm{Tsuc}[\mathrm{ss}$ 公式确定的

其中 $M$ 是尺度最大的原子团中芳香环的个数, $\beta$ 则是描写 2 个相邻 $\pi$ 轨道相互作用的参数,理 论值是 $2.9 \mathrm{eV}$; 但是 Compagnini 等人认为 ${ }^{[8]}$ 在聚合物中 $\beta$ 值以 $2 \mathrm{eV}$ 更加合适. 由此可见芳 香环的聚集数从 $1 \times 10^{14}$ 时的 15 增至 $1 \times 10^{15} \mathrm{~B}^{+} / \mathrm{cm}^{2}$ 时的 30 左右. 这与以前 $\mathrm{X}$ 光电子能 谱(XPS)的研究结果是一致的, 因为 XPS 表明在注人过程中芳香和碳环 $\mathrm{C}-\mathrm{C} / \mathrm{C}=\mathrm{C}$ 相对其 它化学键破坏得较少 ${ }^{[3,4]}$, 而残留下的芳香环和碳环完全可能会凝聚在一起, 形成尺度较大的 原子团以达到更稳定的状态 ${ }^{m}$. 图 2 表明这一过程在从 $1 \times 10^{15}$ 至 $1 \times 10^{16} \mathrm{~B}^{+} / \mathrm{cm}^{2}$ 的注入过 程中会继续下去,这可能是因为改性层将会被进一步碳化,直至在 $1 \times 10^{16} \mathrm{~B}^{+} / \mathrm{cm}^{2}$ 的剂量下形 成尺度较大而又有序度较高的碳环团. 事实上剂量达到 $1 \times 10^{16} \mathrm{~B}^{+} / \mathrm{cm}^{2}$ 时, 改性层的能隙 $E_{8}$ 已几乎不复存在,这意味着聚酰亚胺的大分子结构已被破坏殆尽,同时从能隙上看改性层已与 石墨十分相近;而 XPS 和 Raman 光谱的研究结果 ${ }^{[3]}$ 确也表明: 此时改性层与非晶石墨在短 程有序上存在一定的相似性.

综合上述紫外及可见光谱的结果和有关讨论, 我们认为在离子注人引起的化学键断裂与 重组过程中,随着注人剂量的增加, 聚酰亚胺中的芳香环会逐渐凝聚并形成主要是由共轭键联 接的碳原子组成的原子团, 高能离子注人下的聚酰亚胺将最终演变为类似于无定形石墨的结 构; 离子束表击诱导的这一结构上的重大变化对薄膜电导率的提高具有重要作用.

\section{参考文献}

[ 1] Davenas, J., Xu, X. L., Nucl. Instrum. Methods Phys. Res. B, 1989, 39: 754.

[2] Venkatesan, T., Forrest, S. R. et al., J. Appl. Phys., 1984, 56: 2778.

[3] Xu, D., Xu, X. L., Zou, S. C., Appl. Phys. Letz, 1991, 59: 3110.

[ 4 ] Xu, D., Xu, X. L., Zou, S. C., J. Mazer. Res, 1992, 7: 224.

[5] Tauc, J., Amorphous and Liquid Semiconductors, Plenum, London, 1979.

[ 6] Xu, X. L, Yu, Y. H. et al, Nucl. Instrum. Mcthods Phys. Res. B, 1991, 59/60: 1267.

[ 7 ] Robertson, J. et al., Phys. Rev. B, 1984, 35: 2946.

[8] Compagnini, G., Foti, G. et al., Appl. Phys. Lett., 1990, 57: 2546. 